\title{
THE EFFECTIVENESS OF THE PAEDIATRIC EARLY WARNING TOOL (PEWT) IN IDENTIFYING CHILDREN REQUIRING ADMISSION TO A CRITICAL CARE UNIT
}

\author{
K. O'Loughlin, K. Ruparelia, T. Vince, S. Drysdale, S. Broughton
}

Child Health, Kings College Hospital NHS Foundation Trust, London, UK

Introduction: The Paediatric Early Warning Trigger (PEWT) Tool is used to aid early identification of clinical deterioration and promote effective escalation of care. PEWT is a trigger tool rather than an integer score.

Method: The use of PEWT was prospectively audited during July 2010 at our hospital for all Paediatric admissions, excluding critical care (CCU) or NICU admissions. All observation charts included a PEWT scoring system that assessed 5 physiological parameters. The data were collected twice daily, including demographics, triggering frequency and admission to CCU.

Results: 331 children were admitted (age [range] 6.3 [0.01-18.6] years), 62 (19\%) children triggered. Seven children (all of whom triggered) were admitted to CCU. These seven were more likely to have triggered in more than one time epoch (median [range] number of triggers 3 [1-7] v 0 [0-16], $\mathrm{p}<0.0001$ ). Triggering PEWT detected CCU admission with a sensitivity of $100 \%$ and a specificity of $11 \%$ with a positive predictive value of $100 \%$ and a negative predictive value of $83 \%$. Area under receiver operated curve (AUC) analysis showed that triggering PEWT was highly predictive of CCU admission (AUC 0.91) as was the number of triggering epochs (AUC 0.92).

Conclusion: Triggering PEWT is strongly associated with CCU admission. However, given its low specificity, PEWT is poorly predictive for establishing the need for critical care based on triggering scores alone. Therefore, CCU admission may not be the best measure for evaluating the effectiveness of PEWT, instead effectiveness of interventions would be more appropriate but more difficult to measure. 